Vol.16,\#10, Junio 2009

http://revista-redes.rediris.es

\title{
El rol de las relaciones interpersonales en el proceso de elaboración y puesta en marcha de la política de descentralización en Chile
}

\author{
Carolina Gutiérrez Ruiz - CERAPS (CNRS), Universidad de Lille 2 (Francia)
}

\begin{abstract}
Resumen
En este estudio se analizarán las diferentes fases de elaboración y puesta en marcha de la política de descentralización en Chile. Para ello se han establecido tres fases o secuencias de la política: el decenio que va entre 1960 y 1970, en el cual se identificará la comunidad epistémica que dio origen a la política; luego, el decenio 1980-1990 en el cual se analizará cómo la comunidad logra consolidar sus relaciones y cómo logra emanciparse de las organizaciones que le dieron origen y, finalmente, se establecerá un tercer período que va de 1990 a 1993, en el cual se estudiará cómo la comunidad logra incorporarse en las nuevas estructuras democráticas con el objeto de intervenir en el proyecto de ley de descentralización en Chile.
\end{abstract}

Este método por secuencias permite ampliar la focal de análisis con el objeto de observar no sólo a los actores que forman parte de la red en sus inicios u orígenes sino también aprehender la evolución de la red a través de otros actores que la han ido integrando a través del tiempo y cómo estos nuevos integrantes han podido influenciar el curso que han tomado ciertos eventos.

Palabras clave: Política de descentralización, relaciones interpersonales en el tiempo, Chile.

\begin{abstract}
This article deals with the different stages of production and implementation of the decentralization policy in Chile. Three phases or sequences were established: the decade that goes between 1960 and 1970, in which the epistemic community will be identified to the origin of the policy; then, the decade 1980-1990 in which will be analyzed how the community manages to consolidate relations and how it manages to become emancipated from the original organizations and, finally, in the third period that goes between 1990 and 1993, it will be studied how the community joined to the new democratic structures in order to intervene in the project of law of decentralization in Chile.

This method of sequences allows to extend the focus of analysis in order to observe not just the actors who integrate the policy network in its beginnings but also it allows to depict the evolution of the network through the integration of new actors across the time and how these new members could have influenced the course that certain events have taken.
\end{abstract}

Keywords: Policy of decentralization, interpersonal relations in the time, Chile.

\section{I ntroducción}

Los análisis de políticas públicas han tendido a desestimar el rol jugado por las relaciones interpersonales existentes entre los actores al origen de la elaboración y luego en las diferentes fases de puesta en marcha de dichas políticas. Centrándose, cuando se hace hincapié en los actores, en las trayectorias escolares y profesionales de los mismos, 
dejando en el ámbito de lo implícito el dominio de las relaciones interpersonales existentes entre éstos.

En este sentido, cuando se evoca la existencia de relaciones, los análisis tienden a focalizarse en los actores colectivos o institucionales (Le Galès, Thatcher 1995) para hablar de sus relaciones de colaboración o de competencia, desestimando la importancia de las relaciones existentes entre los actores individuales.

Un concepto interesante que se ha acuñado para rendir cuenta del tema de las relaciones al interior de un grupo, particularmente en lo que respecta al fenómeno de creación de nuevos cuadros cognitivos, es el de comunidad epistémica (Haas 2001). Dichas comunidades reúnen particularmente a científicos, pero también a expertos del sector privado o público que necesitan relacionarse entre sí para entregar nuevas herramientas de análisis frente a problemas complejos, ayudando a las estructuras estatales a definir sus intereses y participando en la construcción de cuadros que delimitan el debate colectivo a través de la propuesta de políticas específicas que luego serán sometidas a negociación.

Dicho concepto permite, además, poner en práctica la teoría constructivista, es decir, pensar las realidades sociales como una construcción, intentando observar cómo el contexto social influye en los comportamientos individuales y por medio de qué mediaciones los límites establecidos por la situación o la estructura definen el universo de lo posible y de lo pensable por los individuos (Lagroye y Al. 2002, p. 15-16) y, al mismo tiempo, analizar cómo los individuos, a través de sus relaciones, influencian y participan en la construcción de este contexto.

A esta corriente se le ha llamado también constructivismo estructuralista: "se piensa en las personas porque ellas son la personificación de posiciones epistemológicas que sólo pueden comprenderse completamente en el campo social en que estas se afirman" (Bourdieu y Al. 1983, p.14) lo que permite definir el aspecto estructural y construido de las relaciones o la interdependencia entre las relaciones sociales y el contexto en que estas se construyen.

Se intentará demostrar en este estudio que las comunidades epistémicas que dan origen a las redes de políticas públicas no son ajenas a las relaciones sociales que pueden establecer y mantener los actores que forman parte de ellas y que participan constantemente de su construcción y recreación.

Para ello se analizará el proceso que permitió la puesta en marcha de una política de descentralización en Chile; abordando, en un primer momento, la estructuración de la comunidad epistémica que dio origen a la red, en donde se analizará que se trata, en sus inicios, de una demanda estatal. Luego, se estudiará el proceso de consolidación de la 
comunidad a través del establecimiento de solidaridades mutuas y de relaciones de colaboración que tomarán una forma polivalente. Finalmente, se analizarán las oportunidades y los limites de la comunidad durante la transición a la democracia en Chile.

Con el objeto de lograr una visión más global del proceso, se ampliará la focal de observación del mismo en tres momentos de la política y no sólo en el momento "fundacional", lo que permite evaluar otras dinámicas que han participado en la política de descentralización y en las cuales otros actores, que no se encontraban en el momento fundacional intervinieron. Se intentará revelar así el rol que pueden adquirir en ciertos momentos y gracias a su inserción en el tejido relacional, actores que sólo han intervenido en una secuencia particular del proceso, pero que con su intervención han participado en la modificación de la red a través del tiempo y con ello en la dirección que han tomado un cierto número de eventos. Para demostrar esto, se han establecido tres momentos o secuencias (Abbott 2001, Pierson, 2004, Desage 2005) de la política de descentralización en Chile: la primera abarca el decenio de 1960-1970, correspondiente al surgimiento de la comunidad estudiada en tanto red fundacional, el segundo momento corresponde al decenio 1980-1990, en donde se analizará la consolidación de las relaciones al interior de la comunidad así como con otros actores y sectores, lo que nos permite hablar de una red de transición; finalmente se estudiará el período entre 1990 y 1993 que corresponde al de la preparación del proyecto de ley de descentralización y a su votación, en donde se analizará cómo la red pasa a la acción gubernamental.

Se podrá concluir así, entre otras cosas, la posición o afiliación múltiple de los miembros de la comunidad en diferentes círculos sociales y en diferentes momentos y cómo ello constituye una oportunidad en la creación de políticas públicas.

\section{Surgimiento y condiciones de posibilidad de las relaciones al interior de la red}

Yo lo que hice fue hacer la conexión entre los especialistas (...) la elaboración programática que hizo esta comisión (de descentralización), es el resumen de muchos años de investigación y de contactos ${ }^{1}$.

Este extracto de una entrevista realizada a uno de los actores que participó durante el período de elaboración y discusión legislativa del proyecto de ley de descentralización en Chile, es decir, el tercer momento estudiado, muestra la existencia de una red preexistente y el conjunto de relaciones que ésta supone.

\footnotetext{
${ }^{1}$ Entrevista personal con la autora, G. M., Santiago de Chile, octubre 2006.
} 
Uno de los primeros interrogantes a que ello conlleva, es buscar el origen de esta red, que este actor define como "años de investigación y de contactos", para luego ver su evolución en el tiempo.

Analizar el surgimiento de la red implica definir los actores que la componen, es decir en nuestro caso, todo aquel que ha participado de la construcción, difusión e implementación de la política de descentralización en Chile. Entendiendo que esta ha sido el fruto de un proceso que encuentra como punto de partida la creación de ODEPLAN (Oficina de Planificación Nacional) en 1965 y como referencia de término, para este análisis, las leyes de municipalidades y de gobierno y administraciones regionales votadas en 1992 y 1993 respectivamente.

Los actores seleccionados como tales responden a dos criterios: primero, han sido definidos como tales a través del método de "bola de nieve" ${ }^{2}$, es decir, por otros actores del proceso así como por ellos mismos, es decir, por medio de su autodefinición como "actor" del proceso ${ }^{3}$. Segundo, los actores retenidos (20) son aquellos que han intervenido en al menos uno de los momentos estudiados, participando así directa o indirectamente en la comunidad epistémica que dio origen a la política en Chile.

La existencia de estas relaciones, así como la cooperación e intervención de los organismos que han participado en la política de descentralización -organismos en los cuales han trabajado los actores retenidos- permite elaborar la hipótesis que indicaría que son las relaciones en el seno de la comunidad epistémica que permiten esta colaboración y la posterior participación de los organismos en la política y no lo contrario.

La visualización de las relaciones entre actores y entre los organismos en las tres secuencias del proceso nos permite establecer dicha hipótesis. En efecto, en el grafo 1 se puede observar una visión de conjunto de los tres momentos (en azul) y de cómo los diferentes actores del proceso (en rojo) participan activamente en uno o varios de esos momentos o secuencias.

\footnotetext{
2 Para censar a esas personas, en tanto actores del proceso, se han utilizado diferentes métodos: primero, se ha realizado una observación participante en ILPES-CEPAL, en 2002, que nos ha permitido establecer en un principio contacto con dos actores del proceso. Estos, a su vez, nos pusieron en contacto o bien mencionaron a otras personas que según ellos respondían a la calificación de actor. Se realizaron tres fases de entrevistas: la primera entre diciembre de 2003 y abril de 2004, la segunda entre octubre de 2005 y enero de 2006, luego entre octubre y diciembre de 2006.

Con el fin de evitar plegarnos exclusivamente a la narración de nuestros entrevistados, lo que podría haber excluido a otros actores con los cuales la relación podría encontrarse en una fase de ruptura, se analizaron igualmente los archivos institucionales (organismos relacionados con la política de descentralización y en los cuales nuestros entrevistados han trabajado y los debates parlamentarios de la ley de descentralización).

${ }^{3}$ Esta información ha sido corroborada por medio de diferentes mecanismos de control: a través de la realización de más de una entrevista por $2 / 3$ de los actores del proceso, la realización de entrevistas a diversos informantes que han trabajado o trabajan en los temas relativos a la descentralización, el estudio de los currículum vitae de los actores (aquellos que los mismos actores nos han entregado y que se adicionan a las informaciones recolectadas en las entrevistas), a los archivos de los organismos en los cuales han trabajado los actores y a los debates parlamentarios de la ley de descentralización.
} 
Por otro lado, en el grafo 2 se puede observar la participación de los organismos (en rojo) a los cuales pertenecen o han pertenecido los actores en las mismas tres secuencias (en azul), estando algunos de ellos en más de una.

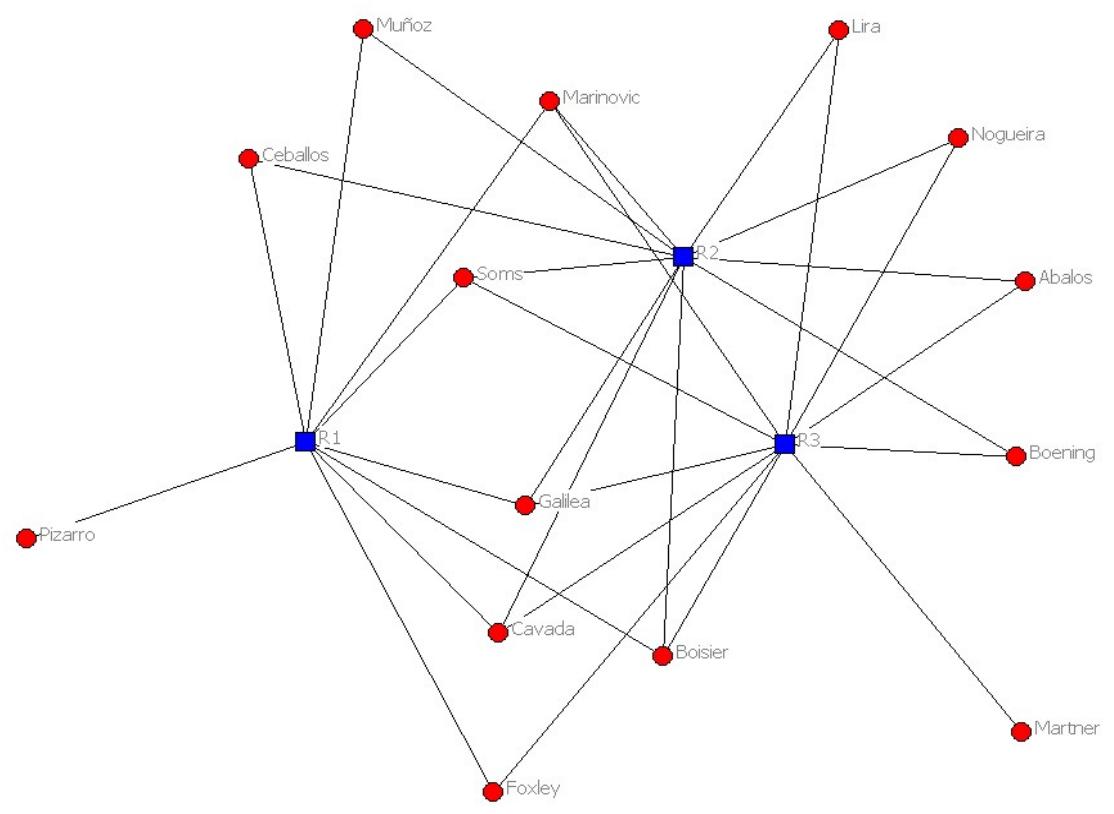

Grafo 1. Red relacional de los actores (en rojo) en las tres secuencias (en azul: R1-R2-R3).

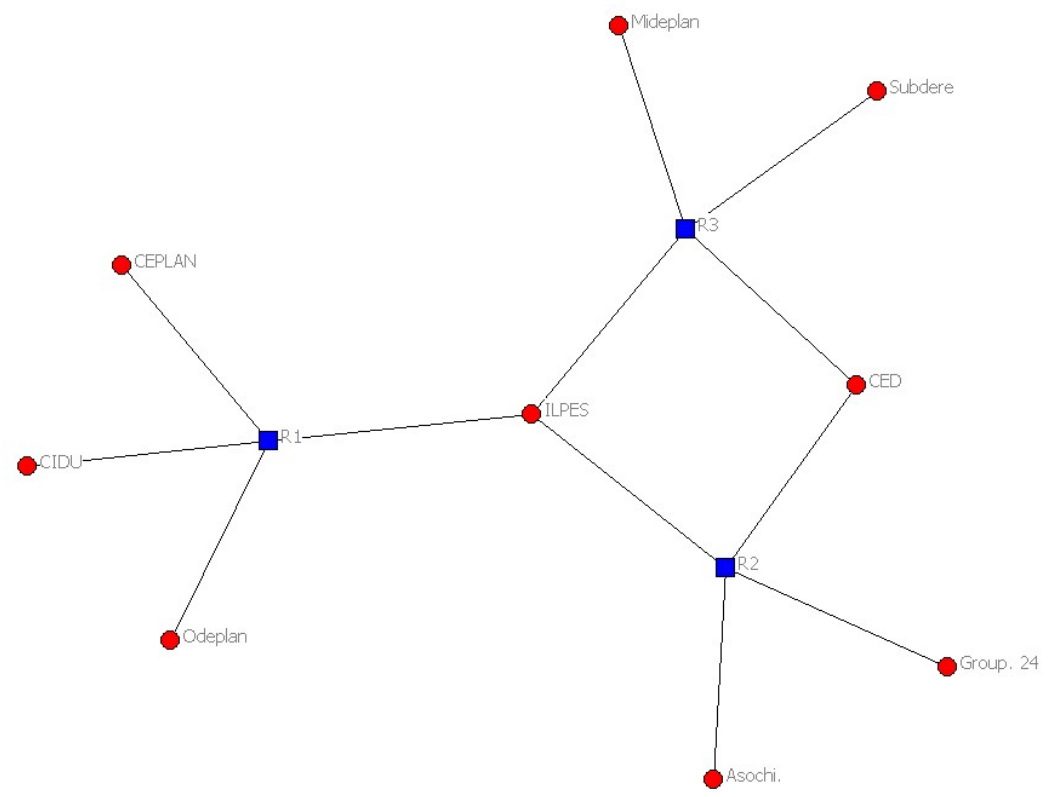

Grafo 2. Red relacional de las instituciones (en rojo) en las tres secuencias (en azul: R1-R2-R3). 
La validación de la hipótesis implica corroborar la existencia de estas relaciones interpersonales así como sus características.

La red estudiada encuentra su momento fundacional con la creación de ODEPLAN (Oficina de Planificación Nacional) por el gobierno demócrata cristiano de Eduardo Frei Montalva (1964-1970).

Este nuevo organismo va a reclutar entre sus miembros a 6 actores - de los 9 estudiados durante esta secuencia- por la mayoría de ellos (5/6) va a tratarse de su primer empleo. Uno de los actores nos describe el clima laboral de la época en el seno de esta institución: “ODEPLAN fue una excelente institución, primero porque había una mística increíble, segundo porque fue una verdadera escuela para muchos de nosotros y en tercer lugar porque había un gran pluralismo político"4

Esta idealización ${ }^{5}$ de la institución podría responder en gran medida a que se trata por estos jóvenes profesionales de su primer empleo y que algunas relaciones que establecerán entre ellos perduraran hasta nuestros días.

ODEPLAN mantendrá desde su creación y hasta 1973, con el golpe de Estado, una relación de colaboración, primero con dos organismos: el ILPES (Instituto Latinoamericano y del Caribe de Planificación Económica y Social), perteneciente a la CEPAL-Naciones Unidas y el CIDU (Centro Interdisciplinario de Desarrollo Urbano), perteneciente a la Facultad de Arquitectura de la Universidad Católica de Chile. Luego, a finales de los años sesenta, con CEPLAN (Centro de Estudios de Planificación Nacional) perteneciente también a la Universidad Católica de Chile.

Los lazos que se establecen entre estos organismos corresponden, en gran medida, a relaciones personales que van a establecer los miembros de dichos organismos (Grafo 3).

En esta perspectiva, se puede decir que dichas relaciones han permitido un trabajo regular que habría facilitado la creación de una comunidad epistémica.

\footnotetext{
${ }^{4}$ Entrevista personal con la autora, S. B., Santiago de Chile, noviembre 2005.

${ }^{5}$ Se habla de idealización pues, por ejemplo, la idea de un "pluralismo político" es bastante relativa, al menos vista con ojos contemporáneos: de los 6 actores, 3 son demócratas cristianos, 2 son socialistas y uno es comunista. Además en esa época la democracia cristiana se situaba, dentro del marco político, en la centroizquierda.
} 


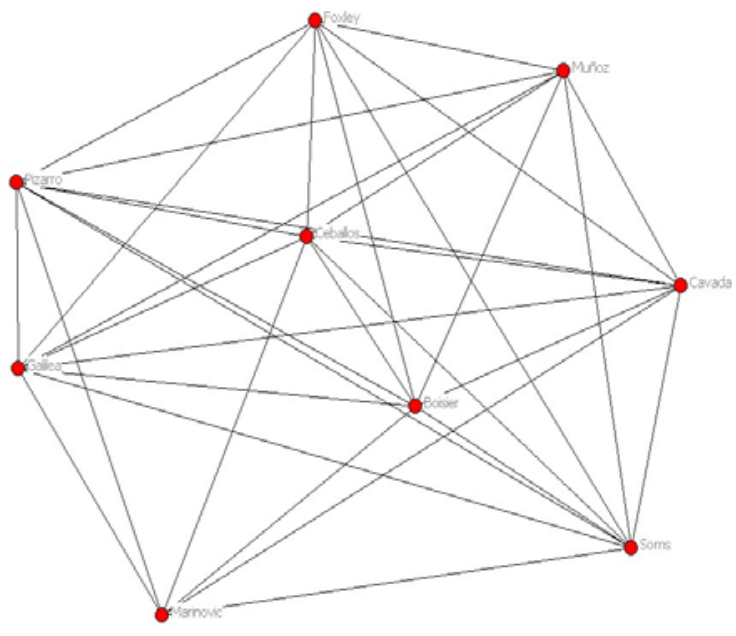

Secuencia 1

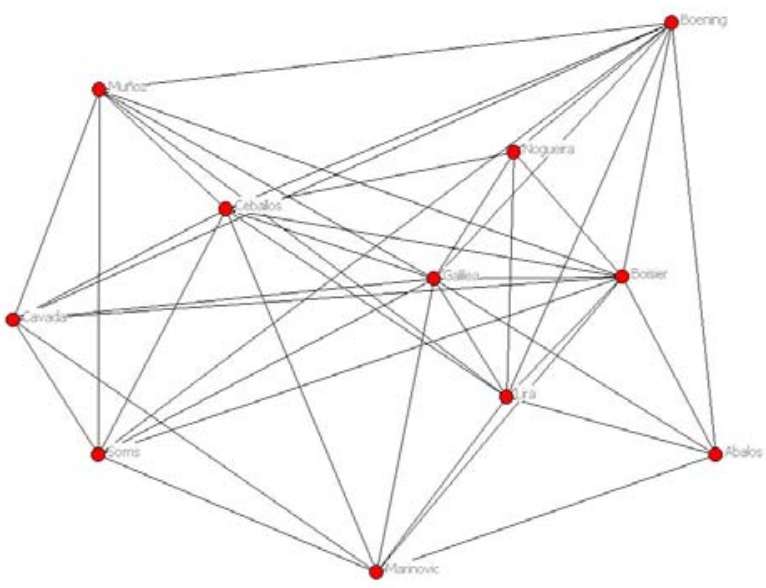

Secuencia 2

Grafo 3. Relaciones interpersonales en la comunidad epistémica en la primera y en la segunda secuencia.

En lo que respecta a la primera secuencia, no son las relaciones interpersonales que preceden a las relaciones ínterorganizacionales, son éstas últimas las que permitieron que los actores pudiesen conocerse y sobre todo, frecuentarse. Sin embargo, estas relaciones lograrán perdurar en el tiempo y sobrepasar el simple estadio de "colegas" en razón de, a lo menos, dos factores: el primero responde a los atributos de los actores y a la homofilia que estos producen. Así, todos los actores son de sexo masculino, todos son militantes de partidos políticos situados en la centro-izquierda e izquierda, todos pertenecen a la misma generación (en la época entre 20 y 30 años), y todos tienen un interés manifiesto por la descentralización como política para ser aplicada en Chile ${ }^{6}$, con una formación profesional común o complementaria ${ }^{7}$. El segundo factor corresponde al cuadro (Goffman 1991) en que estas relaciones se desenvuelven. Se trata de una situación política y social extremadamente tensa, de polarización política y social, en donde el golpe de Estado del 11 de septiembre de 1973 corresponde a su clímax. Este evento, va a generar un nuevo cuadro político que va a consolidar las relaciones entre los actores, ésta vez emancipadas de las relaciones institucionales.

\section{El proceso de consolidación de las relaciones al interior de la red}

Se ha visto que las relaciones de colaboración entre los organismos observados, en la primera secuencia, facilitan los contactos entre actores individuales que podrán, luego, generar dinámicas de trabajo regular dando lugar a la comunidad epistémica.

\footnotetext{
${ }^{6}$ Aunque en la época lo que se entendía por descentralización en esos primero años era en realidad una desconcentración del territorio a través de una política de regionalización. Es sólo a mediados de los años 70 , que el concepto de descentralización entendido como la transferencia de responsabilidades, de autoridad política y administrativa y de recursos, comienza a ser movilizado.

${ }^{7}$ Con una mayoría de economistas, pero contando también con juristas, geógrafos y arquitectos.
} 
La dictadura militar, como situación contextual que marca la segunda secuencia, va a consolidar las solidaridades mutuas entre los actores pertenecientes a la red fundacional, dando lugar a una nueva red que denominaremos de "transición".

Este efecto de contexto va a implicar que entre mediados y finales de los años setenta, todos los actores han abandonado (o han sido obligados a abandonar) ODEPLAN. Ello se explica, por un lado, porque los actores individualmente se oponen al régimen tanto por sus prácticas represivas como por su proyecto ideológico (aplicación de un modelo neoliberal), esto último pudiéndolo evaluar en el seno mismo de la institución y en tanto esta vez que comunidad epistémica, por la política de regionalización puesta en marcha que, en la práctica, no constituye en lo absoluto una descentralización del país ${ }^{8}$. Por otro lado, porque la Democracia Cristiana se sitúa oficialmente en la oposición al régimen, lo que va a implicar que éste partido será considerado como ilegal, sus militantes estando obligados a abandonar sus cargos públicos.

Ello implicará que la institución estatal que hasta aquel momento conformaba la red de organizaciones que pensaban los temas relativos a la aplicación de una política de descentralización en Chile va, en los hechos, cambiar completamente su estrategia y terminar con las colaboraciones preexistentes.

Como la mayoría de los miembros de la comunidad, en lo que concierne a la primera secuencia, trabajaban en esta institución, ello va a significar la búsqueda de nuevas fuentes laborales que respondan a las competencias que hasta ese momento estos actores habían desarrollado y que van a permitirles hacer un vínculo con su trabajo de oposición al régimen dictatorial. Así describe este período uno de los miembros: "éramos amigos, teníamos relaciones, no teníamos una estructura institucional, pero donde podíamos íbamos, cada uno en su espacio, meter el tema (de la descentralización) y después compartíamos opiniones sobre lo que estaba haciendo el gobierno militar, en fin...." . Esta situación de inestabilidad laboral va a incentivar a los miembros de la comunidad a generar espacios organizacionales comunes de trabajo. En esta perspectiva, la red de transición va a crear la ASOCHIPLAN (Asociación Chilena de Planificación) a principios de los años ochenta como un espacio de discusión para generar una nueva y "real" política de descentralización para el país, y también de proyectos de retorno a la democracia, al mismo tiempo que va a investir otros tres organismos: el CED (Centro de Estudios para le Desarrollo), el Grupo de los 24 -organización que tenía por misión principal la elaboración de una propuesta constitucional alternativa a la del régimen- y el ILPES.

\footnotetext{
${ }^{8}$ La regionalización aplicada en Chile en 1975 solo comportaba una desconcentración administrativa. Por lo demás, se van a crear trece regiones a las cuales se les va a designar un número romano (en lugar de su apelación territorial de antiguas provincias).

${ }^{9}$ Entrevista personal con la autora, J.C., Santiago de Chile, noviembre de 2006.
} 
Las dos primeras organizaciones eran eminentemente políticas, con preponderancia de la democracia cristiana. En su seno se crearán - gracias, entre otros, al impulso de los actores pertenecientes a la red de transición- espacios de discusión relativos a la descentralización y que permitirán a nuevos actores entrar en contacto con la red o bien integrarla.

Una parte de estos actores va a aportar una visión comprometida respecto a los temas regionales y de descentralización del país, con competencias desarrolladas en la materia gracias a la realización de masteres y doctorados relacionados con el tema, por lo que van a integrar la red de transición; por otro lado, otro tipo de actores, más involucrados en la esfera política, permitirán que las ideas defendidas por la red puedan ser escuchadas en esta esfera, en el marco de discusiones y charlas respecto a la necesidad de un retorno a la democracia y de las reformas que deberían aplicarse en ese cuadro.

El análisis de estos lazos, contabilizando las tres secuencias, han podido ser caracterizados en diferentes tipos de relaciones (ver anexo), información que ha sido sintetizada en los cuadros 1 y 2 . Básicamente, se ha clasificado al total de relaciones en tres tipos: relaciones profesionales (colegas), relaciones partidarias $\mathrm{y} / \mathrm{o}$ relaciones de amistad.

La información recolectada y analizada permite decir que no todas las relaciones de trabajo tienen por resultado engendrar relaciones de amistad, pero que prácticamente todas las relaciones de amistad tienen relación con el hecho que los actores hayan trabajado conjuntamente. Ello corrobora la idea que el trabajo aumenta, en el caso de los hombres que son mayoritarios dentro de la población activa, las posibilidades de encontrarse y de dialogar (Bidart 1997).

Además, se puede observar una estrecha relación entre las relaciones partidarias y las relaciones de colegas. Ello se explica, en gran medida, porque en Chile los cargos públicos no se obtienen por concurso sino que por expediente, lo que deja un margen de maniobra importante para que otros elementos, más allá de la exclusiva competencia y formación de los candidatos, entren en juego en el momento del reclutamiento. Uno de estos elementos es el de su adscripción partisana. Por lo demás, este modo de funcionamiento se extiende a otras esferas laborales como los centros de estudios.

Al mismo tiempo, existe un cierto número de relaciones polivalentes, nueve en el caso, que son al mismo tiempo de amistad, profesionales y partidarias. 
Finalmente, es interesante constatar que todos los actores de red fundacional se encuentran en la red de transición, a los que se van a sumar nuevos actores, la mayoría de los cuales permanecerán hasta la tercera secuencia. Ello demuestra que las relaciones interpersonales establecidas entre los actores lograron emanciparse de las estructuras institucionales para crear nuevos espacios propios de trabajo y discusión al mismo tiempo que se reapropiaban de otro tipo de organizaciones como los centros de estudio.

\begin{tabular}{|l|c|}
\hline Variables & Frecuencia \\
\hline Relaciones & 128 \\
\hline Profesionales & 116 \\
\hline Amistad & 28 \\
\hline Conocidos & 98 \\
\hline Partidaria & 55 \\
\hline
\end{tabular}

Cuadro 1. Variables relacionales.

\begin{tabular}{|l|c|}
\hline Variables & Frecuencia \\
\hline Amistad-profesional & $28 / 27$ \\
\hline Partidaria-colegas & $43 / 55$ \\
\hline Amistad-colegas-partidaria & 9 \\
\hline
\end{tabular}

Cuadro 2. Relaciones entre las variables.

\section{Los limites de la red o la interdependencia entre las relaciones y el contexto en que estas se desenvuelven}

Se pudo analizar en la parte anterior cómo la red logró adaptarse y crear espacios de discusión y de trabajo en el contexto dictatorial, creando así una nueva dinámica que permitió la consolidación de los lazos entre los miembros que venían de la red fundacional y creando nuevos lazos con quienes van a integrarla en esta secuencia, por lo que se le ha denominado como red de transición. Se verá en esta tercera y última parte del estudio, cómo esta red logrará participar activamente en la tercera secuencia a través de un nuevo proyecto de descentralización del país, por lo que se le llamará red de "acción".

A mediados de los años ochenta la oposición al régimen dictatorial en Chile se une para hacer campaña en el plebiscito en el cual se llamaba a la nación a pronunciarse por ("si") o contra ("no") el mantenimiento del régimen a la cabeza del cuál se encontraba el comandante en jefe del ejército A. Pinochet. El triunfo del "no", permitió abrir una ventana de oportunidad (Kingdon 1995) u oportunidad política (Tarrow 1997), es decir, la posibilidad, para los actores del proceso, de movilizar y valorizar una serie de 
recursos, a los que denominaremos como capital social (Bourdieu 1980), particularmente bajo la forma de reformas políticas.

Esta oportunidad política abrirá paso a un cuadro de transición política gracias al triunfo de la Concertación de partidos por la Democracia ${ }^{10}$ en 1989 , en la cuál el rol de la comunidad epistémica y de su capital social adquiere una relevancia particular. Por recursos se comprende aquí, entonces, a las relaciones personales y contactos mantenidos entre los actores y que les permiten situarse en una posición favorable o de influencia en el momento de negociar el paquete de reformas para ser aplicadas en el período de transición política. Esta transición, es por supuesto una transición a la democracia, y por lo tanto toda reforma que conlleve a una democratización del país (en el discurso o en la realidad práctica) es considerada como prioritaria. En este marco, la reforma de la descentralización, presentada como indispensable para democratizar el país vía la elección de los representantes a nivel subnacional, ocupará una posición privilegiada dentro de la agenda política.

Sin embargo, esta situación favorable no se explica en razón de la dimensión discursiva de dicha reforma (que por lo demás se sitúa dentro de las reformas necesarias a la llamada "modernización del Estado") sino que por las negociaciones que precedieron su incorporación en la agenda.

Dichas negociaciones pasaron por discusiones en torno al tema de redistribuir cuotas de poder entre los diversos actores (antiguos representantes del nivel municipal y con la oposición política para construir un nivel regional), es decir, por consideraciones estrictamente políticas pero en las que los miembros de la comunidad epistémica relacionados con este mundo, bajo la categoría de expertos, podrán ocupar un lugar importante en la definición del proyecto de reforma. Según Gilles Massardier (1996, p.32) « el experto puede definirse como aquél al cual se llama «naturalmente », en un momento dado, porque él posee un saber reconocido por sus pares y por agentes que, en sus actividades, deben aplicar este saber. Siendo este ultimo el fruto de una complejidad cognitiva o de una obligación legal (que es resultado de la construcción social de la complejidad). Dos procesos producen entonces al experto: el reconocimiento de sus pares y el reconocimiento del carácter « indispensable » de su saber más allá del espacio de los especialistas en la materia ».

El hecho de llamar a estas personas, del reconocimiento del cuál éstas pueden beneficiar se explica, además del saber que éstas poseen, por las relaciones que ellas han podido establecer a lo largo de los años y las redes sociales de las cuales son parte. Así los

\footnotetext{
${ }^{10}$ Coalición política de centro izquierda conformada por el Partido Demócrata Cristiano, el Partido Socialista, el Partido por la Democracia y el Partido Radical Social Demócrata.
} 
miembros de la red de transición, llegado el momento de la transición política, se encontraban posicionados en diferentes círculos (profesionales, partidarios y amistosas) que hacían de ellos actores de "pasaje" entre diferentes mundos aumentado sus posibilidades para ser llamados a participar en la elaboración de un proyecto de reforma de este tipo. Prueba de ello es el recuerdo que, de este período, guarda uno de los actores del proceso: "nuestro líder en el grupo, a lo amigo, por edad y por un montón de cosas era S.G., y S. fue nombrado jefe de la División Regional y yo creo (...) que el fuerte de la Subsecretaria de Desarrollo Regional en los primeros cuatro años fue la división regional. Porque las personas que llegamos ahí....Bueno, S. había sido jefe de la Sociedad Interamericana de Planificación, había asesorado a muchos gobiernos en muchas partes, yo había, desde el punto de vista teórico, hecho cursos en la CEPAL, había estudiado el proceso en otras partes con L. L., en Chile en la Universidad Católica, entonces la división regional llegó con diez personas, todas muy competentes, y era la división que tenia el equipo más consolidado, con más experiencia conjunta, entonces.... siento que fuimos el pilar en ese periodo"11.

Ello muestra, por un lado, la interdependencia entre relaciones laborales y afectivas, cuando las primeras son construidas en torno a creencias normativas y, por otra parte, la relación entre políticas públicas y los recursos de los actores que las conciben y las implementan.

La posibilidad de los actores de cambiar las condiciones de lo "posible" responde así a una coyuntura dada o ventana de oportunidad y al conjunto de relaciones que estos tienen en ese momento.

Así cuando G. M. - nuevo actor que intervendrá en la tercera secuencia del proceso dado de su capital político- tuvo que constituir su equipo de trabajo, y sabiendo que él no era considerado como experto en materias de descentralización puesto que no formaba parte de la comunidad epistémica que reflexionaba hasta ese momento sobre el tema, no dudó en llamar personalmente a algunos de los miembros de la red (con los cuales compartía la misma visión política) para formar su equipo de trabajo, los cuales a su vez, llamaron a otros de los miembros de dicha red.

Es este nuevo contexto y las relaciones establecidas por la red de transición, que permitirán a la comunidad epistémica de posicionarse como una red de "acción" en esta tercera secuencia. Como se puede observar en el grafo 4, los miembros que conforman la red de transición se encuentran también, en su casi totalidad en la red de acción,

\footnotetext{
${ }^{11}$ Entrevista personal con la autora, J. A., Santiago de Chile, octubre 2006.
} 
contando como un nuevo miembro a G. M. quien facilitará, gracias a su capital político, la acción de la red en el proyecto de reforma.

Por otro lado, de los nueve actores que conforman la red fundacional, cinco se encuentran en la red de acción, lo que muestra la continuidad y sobre todo la asiduidad del trabajo sobre el tema de la descentralización que venía desarrollando la red desde hacia casi tres décadas.

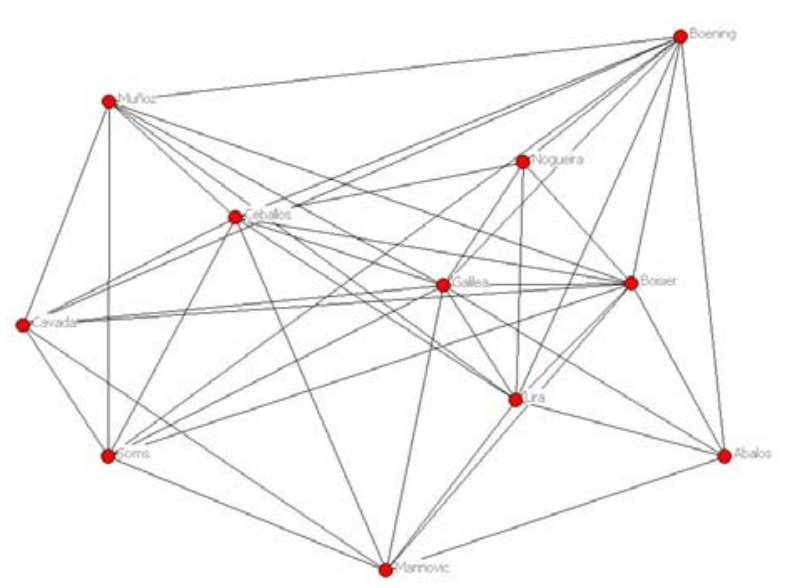

Secuencia 2

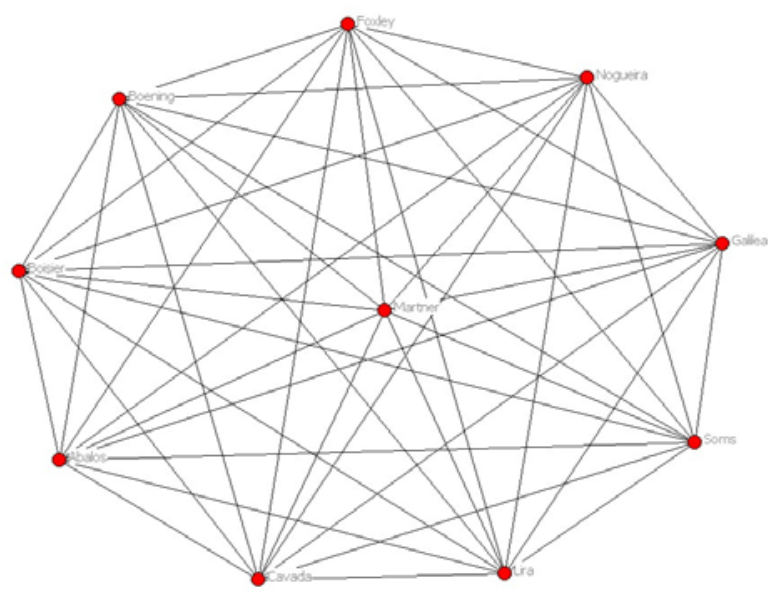

Secuencia 3

Grafo 4. Relaciones interpersonales en la comunidad epistémica en la segunda y en la tercera secuencia.

El nuevo contexto político no creará, sin embargo, sólo "nuevas oportunidades". La posibilidad de poner en práctica un proyecto sobre el cuál los actores reflexionaban desde hacía décadas va a conllevar también a ciertas rupturas de relación que se explican, en la mayoría de los casos, por divergencias cognitivas sobre los instrumentos más apropiados para llevar a buen término el proyecto de descentralización, pero sobre todo sobre ciertas atribuciones institucionales. Lo que corrobora, en este caso, que las creencias y el aspecto profesional se encuentran íntimamente ligados cuando se trata de personas convencidas sobre lo bien fundado de sus proyectos reformistas, sobre todo cuando dichos proyectos amalgaman creencias normativo-epistemológicas y creencias ideológicas.

\section{Conclusión}

Se ha visto que las relaciones de colaboración entre los organismos observados, en la primera secuencia, facilitan los contactos entre actores individuales que pudieron, luego, generar dinámicas de trabajo regular dando lugar a la comunidad epistémica. La dictadura militar consolidó las solidaridades mutuas entre los actores pertenecientes a la red fundacional, dando lugar a una nueva red que denominamos de transición. En efecto, esta logró adaptarse y crear espacios de discusión y de trabajo en el contexto dictatorial, 
estableciendo así una nueva dinámica que permitió la consolidación de los lazos entre los miembros que venían de la red fundacional y creando nuevos lazos con quienes la integraron en la segunda secuencia. Finalmente, se analizó cómo la red logró participar activamente en la tercera secuencia a través de un nuevo proyecto de descentralización del país, por lo que se le denominó red de acción.

Efectivamente, el estudio ha buscado mostrar que los expertos, que conforman la comunidad epistémica y que son llamados en un momento dado para participar de los proyectos de reforma, no son sólo necesarios por sus conocimientos sino que por el reconocimiento del capital social que éstos poseen. Dicho capital se forja durante años de trabajo intelectual, pero también de relaciones amistosas no sólo en el seno de una comunidad epistémica en particular sino que con otras esferas sociales, como la política.

Se ha intentado mostrar así que son las relaciones interpersonales las que facilitan los contactos organizacionales, y sobre todo, su continuación en el tiempo. Ello se explica también por la capacidad de los actores a adaptarse a las diferentes situaciones de contexto y a crear oportunidades políticas.

Cuando se observa la red en el tiempo y el sistema de relaciones que se establece, se puede constatar, por ejemplo, el carácter marginal de la variable generacional frente a otras variables como la militancia o el trabajo en conjunto, estas dos últimas correspondientes a variables relacionales más que a los atributos individuales de los actores. Al mismo tiempo, ambas variables parecen tener una estrecha relación en el origen de las relaciones amistosas.

En definitiva, se ha intentado demostrar la importancia que pueden tener las relaciones interpersonales en la elaboración y aplicación de políticas públicas. Ello conlleva a cuestionar los criterios que tienden a neutralizar aspectos que no formarían parte de la "competencia" estricta, entendida como un saber o conocimiento, de quienes piensan y ponen en marcha dichas políticas.

\section{Bibliografía}

Abbott, A. (2001). Time matters: On theory and method (Chicago, London: The University of Chicago Press).

Bès, M.-P., Grossetti, M. (2001). «Encastrements et découplages dans les relations science industrie », Revue Française de Sociologie, 42 (2) : 327-355.

Bidart, C. (1997). L'amitié, un lien social (Paris : La Découverte, 1997).

Bourdieu, P. (1980). «Le capital social », Actes de la recherche en sciences sociales, 31 (1), <http://www.persee.fr/showPage.do?urn=arss_0335-5322_1980_num_31_1_2069>. 
Bourdieu, P., Chamboredon, J.-C., Passeron, J.-C. (1983), primera edición 1968, Le métier de sociologue (Paris-La Haye : École des Hautes Etudes en Sciences Sociales et Mouton éditeur).

De Federico de la Rúa, A. (2005). "Amistad e identificación: las micro fundaciones de las pertenencias macro. Amigos europeos e identidad europea' », REDES. Revista Hispana para el Análisis de Redes Sociales. Volumen especial : 19-50.

Desage, F. (2005). Le 'consensus' communautaire contre l'intégration intercommunale: séquences et dynamiques d'institutionnalisation de la communauté urbaine de Lille (1964-2003), Thèse pour le doctorat en science politique, Université de Lille 2.

Ferrand, A., de Federico de la Rua, A. (2006). "Méthodes d'analyse des réseaux sociaux », 459-495, in Caselli, Graziella, Vallin, Jacques, Wunsch, Guilleaume (éd.), Encyclopédie de démographie: analyse et synthèse. (VII), Observations, méthodes auxiliaires et enseignement (Paris : INED).

Gil-Mendieta, J., Ruiz León, A. (2004). “redes variables en el tiempo: visualización con Pajek", Redes. Revista Hispana para el Análisis de Redes Sociales, 6 (4). Consulta [2003-2008] en <http///revista-redes. rediris.es>.

Goffman, E. (1991). 1a ed. 1974, Les cadres de l'expérience, (Paris, Minuit).

Haas, P. (2001). "Policy Knowledge: Epistemic Communities", en N. J. Smelser and P. B. Baltesm (eds.), International Encyclopedia of the Social and Behavioral Sciences (Amsterdam: Elsevier).

Kingdon, J.W. (1995). Agendas, alternatives and public policies (New York: Longman). Lagroye, J. (2002). sociologie politique (Paris: Presses de Sciences po, Dalloz).

Lazega, E. (1998). Réseaux sociaux et structures relationnelles (Paris: PUF, Que saisje ?).

Lazega, E. y Lebeaux (1995). «Capital social et contrainte latérale », Revue Française de Sociologie, 36 (1) : 759-777.

Le Galès, P., Thatcher, M. (1995). Les réseaux de politique publique. Débat autour des policy networks (Paris: L'Harmattan).

Lozares Colina, C. (y Al.) (2006). “Prólogo. Del atomismo al relacionismo: la red sociocognitiva como paradigma de cambio en la concepción de lo social y de la cognición", Redes. Revista Hispana para el Análisis de Redes Sociales, 10 (1). Consulta [28-03-2008] en $<$ http///revista-redes. rediris.es $>$.

Massardier, G. (1996). Expertise et aménagement du territoire : I'Etat savant (Paris : L'Harmattan). 
Pierson, P. (2004). Politics in time: History, institutions and social analysis (Princeton and Oxford: Princeton University Press).

Pizarro, N. (2000). "Regularidad social, redes de lugares y reproducción social", Política y Sociedad, 33: 167-198, Consulta en

<http://www.ucm.es/BUCM/revistasBUC/portal/modules. php?name=Revistas2\&id=POSO>.

Simmel, G. (1999). $1^{\text {a }}$ ed. 1908. Sociologie: Etudes sur les formes de la socialisation (Paris: PUF).

Tarrow, S. (1997). 1a edición inglesa 1994, El poder en movimiento: Los movimientos sociales, la acción colectiva y la política (Madrid: Alianza Editorial).

White, H. (2000). «La construcción de las organizaciones sociales como redes múltiples", Política y Sociedad, 33: 97-104. Consulta en

<http://www.ucm.es/BUCM/revistasBUC/portal/modules. php?name=Revistas2\&id=POSO $>$. 


\section{Anexo Metodológico}

\begin{tabular}{|c|c|c|c|c|c|c|}
\hline Ego & Identifica & Relación & & & & \\
\hline & Alter & colegas & amistad & simetría/ alter & conocidos & partidaria \\
\hline 1 & 2 & 1 & & & 1 & \\
\hline 1 & 3 & 1 & 1 & 1 & & \\
\hline 1 & 4 & 1 & & & 1 & \\
\hline 1 & 6 & 1 & 1 & 1 & & 1 \\
\hline 1 & 8 & 1 & & & 1 & \\
\hline 1 & 10 & 1 & 1 & 1 & & \\
\hline 1 & 11 & 1 & & & 1 & \\
\hline 1 & 12 & 1 & & & 1 & \\
\hline 1 & 13 & 1 & & & 1 & \\
\hline 1 & 14 & 1 & & & 1 & \\
\hline 1 & 15 & 1 & & & 1 & \\
\hline 1 & 16 & 1 & & & 1 & \\
\hline 1 & 19 & 1 & & & 1 & \\
\hline 1 & 20 & 1 & 1 & 1 & & \\
\hline 2 & 3 & 1 & & & 1 & \\
\hline 2 & 6 & & & & 1 & 1 \\
\hline 2 & 7 & & & & 1 & 1 \\
\hline 2 & 8 & & & & 1 & 1 \\
\hline 2 & 9 & & & & 1 & 1 \\
\hline 2 & 10 & 1 & 1 & 1 & & \\
\hline 2 & 11 & 1 & & & 1 & \\
\hline 2 & 19 & & & & 1 & 1 \\
\hline 3 & 4 & 1 & 1 & 1 & & \\
\hline 3 & 5 & 1 & 1 & 1 & & 1 \\
\hline 3 & 6 & 1 & 1 & 1 & & 1 \\
\hline 3 & 7 & 1 & 1 & 1 & & \\
\hline 3 & 8 & 1 & & & 1 & \\
\hline 3 & 10 & 1 & 1 & 1 & 1 & 1 \\
\hline 3 & 11 & 1 & 1 & 1 & & \\
\hline 3 & 12 & 1 & & & 1 & 1 \\
\hline 3 & 13 & 1 & & & 1 & 1 \\
\hline 3 & 14 & 1 & & & 1 & 1 \\
\hline 3 & 15 & & & & 1 & 1 \\
\hline 3 & 16 & 1 & & 2 & & 1 \\
\hline 3 & 17 & 1 & & & 1 & 1 \\
\hline 3 & 18 & 1 & & & 1 & \\
\hline 3 & 20 & 1 & & & 1 & \\
\hline 4 & 5 & 1 & 1 & 1 & & \\
\hline 4 & 6 & 1 & & & 1 & 1 \\
\hline 4 & 7 & 1 & & & 1 & 1 \\
\hline 4 & 8 & 1 & 1 & 1 & & 1 \\
\hline 4 & 10 & 1 & & & 1 & \\
\hline 4 & 11 & 1 & 1 & 1 & & \\
\hline 4 & 12 & 1 & & & 1 & \\
\hline 4 & 13 & 1 & 1 & 1 & & \\
\hline 4 & 15 & 1 & & & 1 & \\
\hline 4 & 16 & 1 & & & 1 & \\
\hline
\end{tabular}




\begin{tabular}{|c|c|c|c|c|c|c|}
\hline 4 & 17 & 1 & & & 1 & \\
\hline 4 & 18 & 1 & & & 1 & 1 \\
\hline 4 & 19 & 1 & & & 1 & 1 \\
\hline 5 & 7 & 1 & 1 & 1 & & \\
\hline 5 & 10 & & 1 & 1 & & 1 \\
\hline 5 & 11 & 1 & 1 & 1 & & \\
\hline 5 & 12 & 1 & & & 1 & 1 \\
\hline 5 & 13 & 1 & 1 & 1 & & 1 \\
\hline 5 & 14 & & & & 1 & 1 \\
\hline 5 & 15 & 1 & & & 1 & 1 \\
\hline 5 & 16 & & & & 1 & 1 \\
\hline 5 & 17 & 1 & & & 1 & 1 \\
\hline 5 & 18 & 1 & & & 1 & \\
\hline 6 & 7 & 1 & 1 & 1 & & 1 \\
\hline 6 & 8 & 1 & & & 1 & \\
\hline 6 & 9 & 1 & & & 1 & \\
\hline 6 & 10 & 1 & 1 & 1 & & \\
\hline 6 & 11 & 1 & & & 1 & \\
\hline 6 & 12 & 1 & & & 1 & \\
\hline 6 & 13 & 1 & & & 1 & \\
\hline 6 & 14 & 1 & & & 1 & \\
\hline 6 & 15 & 1 & & & 1 & \\
\hline 6 & 16 & 1 & & & 1 & \\
\hline 6 & 17 & 1 & & & 1 & 1 \\
\hline 6 & 18 & 1 & & & 1 & 1 \\
\hline 6 & 19 & 1 & & & 1 & 1 \\
\hline 7 & 9 & & & & 1 & 1 \\
\hline 7 & 10 & 1 & 1 & 1 & & \\
\hline 7 & 11 & 1 & 1 & 1 & & \\
\hline 7 & 16 & 1 & & & 1 & \\
\hline 8 & 9 & 1 & & & 1 & 1 \\
\hline 8 & 10 & 1 & 1 & 1 & & \\
\hline 8 & 11 & 1 & & & 1 & \\
\hline 8 & 12 & 1 & & & 1 & \\
\hline 8 & 13 & 1 & & & 1 & \\
\hline 8 & 14 & 1 & & & 1 & \\
\hline 8 & 15 & 1 & & & 1 & \\
\hline 8 & 16 & 1 & & & 1 & \\
\hline 8 & 19 & 1 & & & 1 & 1 \\
\hline 9 & 4 & 1 & & & 1 & 1 \\
\hline 9 & 12 & 1 & & & 1 & \\
\hline 9 & 13 & 1 & & & 1 & \\
\hline 9 & 14 & 1 & & & 1 & \\
\hline 9 & 15 & 1 & & & 1 & \\
\hline 9 & 19 & 1 & & & 1 & 1 \\
\hline 9 & 20 & 1 & & & 1 & \\
\hline 10 & 11 & 1 & & 2 & & \\
\hline 10 & 12 & 1 & & & 1 & 1 \\
\hline 10 & 13 & 1 & & & 1 & 1 \\
\hline 10 & 14 & 1 & & & 1 & 1 \\
\hline 10 & 15 & 1 & & & 1 & 1 \\
\hline 10 & 16 & 1 & & & 1 & 1 \\
\hline
\end{tabular}




\begin{tabular}{|c|c|c|c|c|c|c|}
\hline 10 & 17 & 1 & & 2 & & 1 \\
\hline 10 & 18 & 1 & & & 1 & \\
\hline 10 & 19 & 1 & & & 1 & \\
\hline 10 & 20 & 1 & & & 1 & \\
\hline 11 & 8 & 1 & & & 1 & \\
\hline 11 & 12 & 1 & & & 1 & 1 \\
\hline 11 & 13 & 1 & 1 & & & \\
\hline 11 & 14 & 1 & & & 1 & \\
\hline 11 & 15 & 1 & & & 1 & \\
\hline 11 & 16 & 1 & & & 1 & \\
\hline 11 & 17 & 1 & & & 1 & \\
\hline 11 & 18 & 1 & & & 1 & \\
\hline 12 & 13 & 1 & & & 1 & 1 \\
\hline 12 & 14 & 1 & 1 & & & 1 \\
\hline 12 & 15 & 1 & 1 & & & 1 \\
\hline 12 & 16 & 1 & & & 1 & 1 \\
\hline 12 & 19 & 1 & & & 1 & \\
\hline 13 & 14 & 1 & & & 1 & 1 \\
\hline 13 & 15 & 1 & & & 1 & 1 \\
\hline 13 & 16 & 1 & & & 1 & 1 \\
\hline 13 & 17 & & & & 1 & 1 \\
\hline 13 & 18 & 1 & & & 1 & \\
\hline 13 & 19 & 1 & & & 1 & \\
\hline 14 & 15 & 1 & & & 1 & 1 \\
\hline 14 & 16 & 1 & & & 1 & 1 \\
\hline 14 & 19 & 1 & & & 1 & \\
\hline 15 & 16 & 1 & & & 1 & 1 \\
\hline 15 & 17 & & & & 1 & 1 \\
\hline 17 & 18 & 1 & 1 & 1 & & \\
\hline \multicolumn{7}{|l|}{ Media } \\
\hline Suma & 127 & 116 & 28 & & 98 & 55 \\
\hline
\end{tabular}

